\title{
Tissue diagnosis of intestinal microsporidiosis using a fluorescent stain with Uvitex $2 \mathrm{~B}$
}

\author{
C Franzen, A Müller, B Salzberger, G Fätkenheuer, S Eidt, G Mahrle, V Diehl, \\ M Schrappe
}

\begin{abstract}
Aims-To detect intestinal microsporidiosis in paraffin wax embedded biopsy specimens using a fluorescence technique incorporating optical brighteners.

Methods-Eight HIV infected patients with confirmed intestinal microsporidiosis (six with Enterocytozoon bieneusi, one with Encephalitozoon intestinalis and one with Encephalitozoon cuniculi infection) and 10 without infection were studied. Tissue sections of paraffin wax embedded duodenal biopsy specimens were stained with $1 \%$ Uvitex $2 \mathrm{~B}$, coded and analysed independently by two investigators.

Results-In all eight cases with confirmed intestinal microsporidian infection, spores could be detected easily in tissue sections using the fluorescence technique. Spores or other elements consistent with microsporidiosis were not found in the 10 patients without infection.

Conclusion-Staining of tissue sections from paraffin wax embedded intestinal biopsy specimens with stains incorporating Uvitex $2 B$ is a rapid and easy technique for the diagnosis of intestinal microsporidiosis.

(f Clin Pathol 1995;48:1009-1010)
\end{abstract}

Keywords: Uvitex 2B, microsporidiosis, HIV.

Chronic diarrhoea is a common symptom in patients infected with HIV and is often caused by infection with microsporidia. ${ }^{12}$ Three different microsporidian species, Enterocytozoon bieneusi, Encephalitozoon intestinalis, and recently Encephalitozoon cuniculi, have been recognised in intestinal biopsy specimens of HIV infected patients. ${ }^{1-3}$

Histological diagnosis of intestinal microsporidiosis in paraffin wax embedded material using routine stains is difficult because of the small size and variable staining characteristics of the parasites. ${ }^{145}$ Intestinal microsporidiosis can be diagnosed in paraffin wax embedded material using special stains, but most of these stains are time consuming to apply and are not suitable for routine use. ${ }^{4-6}$ As a result, tissue diagnosis depends heavily on electron and light microscopy of plastic or resin embedded tissue; these techniques, however, are not widely available. ${ }^{17-9}$

Microsporidian spores can be detected in stool samples and touch preparations of intestinal biopsy specimens using fluorescent stains with optical brightening agents such as Uvitex $2 \mathrm{~B} .{ }^{9-12}$ Here, we report the use of a fluorescent stain incorporating Uvitex 2B for the diagnosis of intestinal microsporidiosis in tissue sections of paraffin wax embedded intestinal biopsy specimens from patients with AIDS.

\section{Methods}

Eighteen patients infected with HIV (all CDC C3) were included in the study. Eight patients had intestinal microsporidiosis, caused by Enterocytozoon bieneusi in six, Encephalitozoon intestinalis in one and Encephalitozoon cuniculi in one, diagnosed by stool examination using a fluorescence technique incorporating Uvitex $2 \mathrm{~B}^{910}$ and confirmed by transmission electron microscopy of intestinal biopsy specimens using standard procedures. ${ }^{37}$ The other 10 patients had no microsporidian spores on examination of their stool samples and no parasites in intestinal biopsy specimens examined using transmission electron microscopy.

Two biopsy specimens from each patient were obtained from the distal duodenum using a flexible fiberglass endoscope. One biopsy specimen was fixed in $10 \%$ neutral buffered formalin and embedded in paraffin wax for light microscopy without prior orientation. Tissue sections, 3 to $4 \mu \mathrm{m}$ thick, were stained with a fluorescent stain as described by van Gool et al for staining microsporidia in stool samples. ${ }^{10}$ Tissue sections were deparaffinised using $\mathrm{xy}-$ lene and rehydrated through graded ethanol. Slides were fixed in methanol for two minutes and stained with $1 \%$ Uvitex $2 \mathrm{~B}$ in phosphate buffered saline (PBS) for 30 minutes, washed in PBS for a few seconds, counterstained with $0.5 \%$ Evan's blue in PBS for 60 seconds, dehydrated through graded ethanol, and embedded in eukitt. Slides were coded and analysed independently by two investigators under a Zeiss fluorescence micrascope with a $50 \mathrm{~W}$ mercury high pressure lamp and an excitation filter with a transmission range from 355 to $425 \mathrm{~nm}$ and a suppression filter of $460 \mathrm{~nm}$. The other biopsy specimen was fixed in $3 \%$ glutaraldehyde and processed for transmission electron microscopy using a Zeiss EM 10 as described previously. ${ }^{7}$

\section{Results}

In all eight cases with a confirmed intestinal microsporidian infection, spores could be easily detected by both investigators in tissue sections 


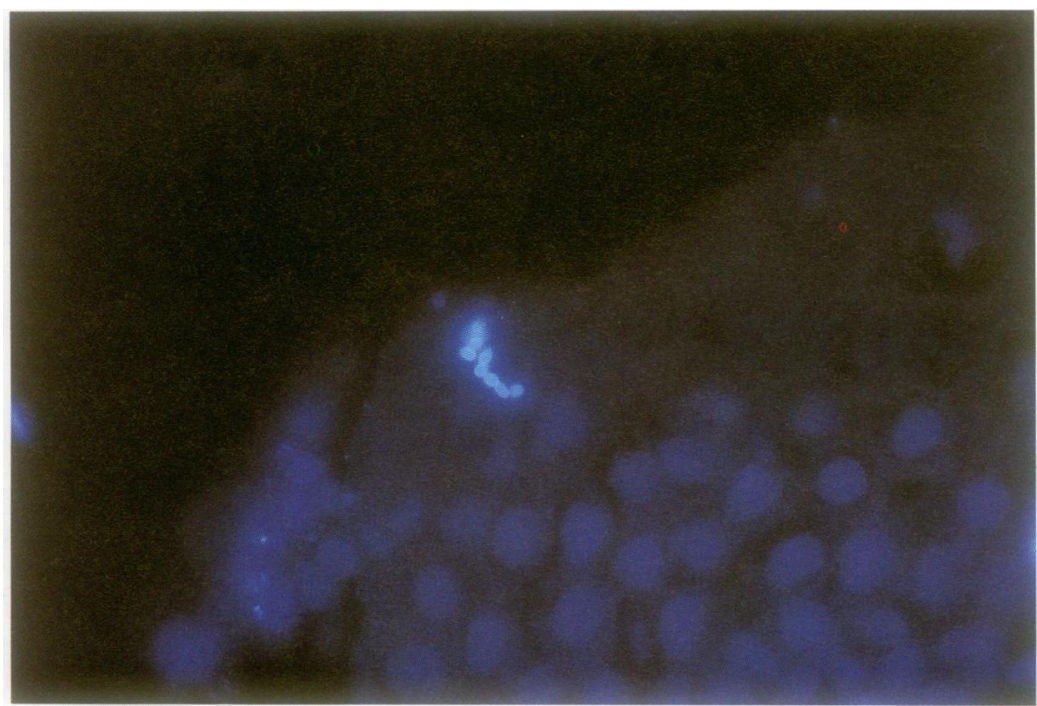

Tissue section of a paraffin wax embedded intestinal biopsy specimen with spores of Enterocytozoon bieneusi in duodenal enterocytes. Fluorescent stain with Uvitex $2 B$, original magnification $\times 1000$. sensitivity and specificity of most of these stains are unknown and some of them are time consuming to apply and are unsuitable for routine use. Fluorescein tagged anti-Encephalitozoon antibodies are useful, especially for differentiating between species, but are not widely available; anti-Enterocytozoon antibodies have yet to be developed. ${ }^{2}$ As a result, tissue diagnosis of intestinal microsporidiosis depends heavily on electron microscopy of plastic or resin embedded tissue. ${ }^{178}$

Fluorescent stains incorporating optical brightening agents such as Uvitex $2 \mathrm{~B}$ have been described for staining microsporidian spores in stool samples, body fluids and touch preparations of biopsy specimens, the latter requiring fresh material..$^{9-12}$ The application of these stains to paraffin wax embedded material would allow intestinal microsporidiosis to be diagnosed routinely without recourse to special staining techniques and would also be useful for epidemiological research.

Some studies found that examination of stool samples is more sensitive compared with histological examination of intestinal biopsy specimens ${ }^{10}$ but we found no such difference in sensitivity in this study.

In conclusion, staining of tissue sections of paraffin wax embedded intestinal biopsy specimens with fluorescent stains incorporating Uvitex $2 \mathrm{~B}$ is a rapid and easy technique for the diagnosis of intestinal microsporidiosis.

1 Orenstein JM, Chiang J, Steinberg W, Smith PD, Rotterdam $H$, Kotler DP. Intestinal microsporidiosis as a cause of diarrhea in human immunodeficiency virus-infected patients: a report of 20 cases. Hum Pathol 1990;21:475-81.

patients: a report of 20 cases. Hum Pathol 1990;21:475-81.
2 Weber R, Bryan RT, Schwartz DA, Owen RL. Human microsporidial infections. Clin Microbiol Rev 1994;7:426-

61 . structures. Some of the spores had a concave inner stripe or shadow, as described previously in stool samples by van Gool et al. ${ }^{9}$ Spores were present in clusters in the apical part of intestinal enterocytes and in the two cases with $E$ intestinalis and $E$ cuniculi infection, were also present in lamina propria cells.

The size of the spores enabled us to differentiate between $E$ bieneusi and Encephalitozoon spores but not between $E$ intestinalis and $E$ cuniculi.

\section{Discussion}

Diagnosis of intestinal microsporidiosis is difficult because of the small size and variable staining characteristics of the parasites. ${ }^{5}$ Highly experienced pathologists have reliably and consistently identified microsporidia in tissue sections using routine staining techniques such as haematoxylin and eosin or Giemsa, but sensitivity and specificity is low, especially in the case of low parasite load. ${ }^{15}$ Different stains such as a modified Gomori trichrome stain, ${ }^{4}$ modified Gram stains (Brown-Benn, Brown-Hopps), the Warthin-Starry stain, ${ }^{6}$ or immunofluorescent stains ${ }^{2}$ can be used to diagnose microsporidiosis in paraffin wax embedded material on light microscopy. The ranzen C, Schwartz DA, Visvesvara GS, Müller A, Schwenk A, Salzberger B, et al. Disseminated antibody confirmed Encephalitozoon cuniculi with asymptomatic infection of the gastrointestinal tract in a patient with AIDS. Clin Infect Dis 1995 (in press). diagnosis of intestinal microsporidiosis using the Chromotrope-2R modified trichrome stain. Arch Pathol Lab Med 1993;117:1249-51.

5 Kotler DP, Giang TT, Garro ML, Orenstein JM. Light microscopic diagnosis of microsporidiosis in patients with AIDS. Am $₹$ Gastroenterol 1994;89:540-4.

6 Field AS, Mariott DJ, Hing MC. The Warthin-Starry stain in the diagnosis of small intestinal microsporidiosis in in the diagnosis of small intestinal microsporidiosis in HIV-in

7 Franzen C, Fätkenheuer G, Salzberger B, Müller A, Mahrle $\mathrm{G}$, Diehl $\mathrm{V}$, et al. Intestinal microsporidiosis in patients with acquired immunodeficiency syndrome - report of three more German cases. Infection 1994;22:417-19.

8 Peacock CS, Blanshard C, Tovey DG, Ellis DS, Gazzard BG. Histological diagnosis of intestinal microsporidiosis in patients with AIDS. $\mathcal{f}$ Clin Pathol 1991;44:558-63.

9 van Gool T, Snijders F, Reiss P, Eeftinck Schattenkerk JKM, van den Berg Weerman MA, Bartelsman JFWM, et al.
Diagnosis of intestinal and disseminated microsporidial Diagnosis of intestinal and disseminated microsporidial infections in patients with HIV by a new rap

10 van Gool T, Canning EU, Dankert J. An improved practical and sensitive technique for the detection of microsporidian and sensitive technique for the detection of microsporidian
spores in stool samples. Trans $R$ Soc Trop Med Hyg 1994; 88:189-90.

11 Vára J, Dahbiová R, Hollister WS, Canning EU. Staining of microsporidian spores by optical brighteners with remarks on the use of brighteners for the diagnosis of AIDS associated human microsporidiosis. Folia Parasitol (Praha) 1993;40:267-72.

12 Vára j, Nohynková E, Machala L, Spála J. An extremely rapid method for detection of microsporidia in biopsy materials from AIDS patients. Folia Parasitol (Praha) 1993;40:273-4.
4 Giang TT, Kotler DP, Garro ML, Orenstein JM. Tissue 